\title{
TECHNICAL COMMENTARY
}

\section{SVT Discrimination Features in St. Jude Medical ICDs}

TIM MCINTYRE, MS

St. Jude Medical, St. Paul, MN

\author{
ISSN 2156-3977 (print) \\ ISSN 2156-3993 (online) \\ (C) 2016 Innovations in Cardiac \\ Rhythm Management
}

\section{Poghosyan, et al.: Inappropriate ICD Shocks as a Consequence of a Refinement in SVT Discrimination Algorithms}

The SVT Discrimination features in St. Jude Medical ICDs are customizable to provide high specificity and sensitivity in the presence of complex arrhythmias. One of the programmable parameters is the AV Interval Delta. This parameter can be useful in the discrimination of dual tachycardias by calculating AV Dissociation in the V=A Rate Branch. When active, the algorithm examines AV intervals in a sliding window (window $=16$ intervals in this case) during classification of tachycardia intervals. The difference between the second longest and second shortest AV intervals is calculated. Rejecting the longest and shortest AV intervals prevents isolated beats from forcing a VT diagnosis. If this calculated difference is greater than or equal to the programmed AV Interval Delta (60 ms in this case), then AV Dissociation is declared and a diagnosis of VT rather than SVT will occur as in Figure 4.

In this case the combination of a slow VT zone of $123 \mathrm{bpm}$, a PVC during tachycardia and the AV Interval Delta parameter value of $60 \mathrm{~ms}$ led to a diagnosis of VT resulting in inappropriate therapies. Based on the detailed arrhythmia data provided by the device diagnostics, the device was re-programmed to avoid this in the future.

While not all of the $16 \mathrm{AV}$ intervals were annotated in the Marker Channel due to $\mathrm{P}$ waves falling into the PVARP, the AV Interval Delta was still applied to those intervals as part of the standard function of the algorithm. The specific values for those intervals are available in the device Session Records, which is an encrypted file saved on the St. Jude programmer each time a device is interrogated. The EGM strips were clipped from Acrobat PDF files that are saved on the St. Jude programmer during printing.

\footnotetext{
Timothy G. McIntyre, MS is an employee of St. Jude Medical, Inc. Authors invited the commentary in order to provide perspectives on optimal programming protocols for the device.

Manuscript received March 16, 2016, Final version accepted April 19, 2016.

Address correspondence to: Tim McIntyre, Senior EP Tech Service Specialist, One St. Jude Medical Drive, St. Paul, MN, 55117.

E-mail: TMcIntyre@sjm.com
} 